\title{
基于双高斯分层表面理论的机械密封研究综述
}

\author{
胡松涛 ${ }^{1}$ 黄伟峰 $^{2}$ 史 熙 ${ }^{1}$ 彭志科 $^{1}$ 刘向锋 $^{2}$ \\ (1. 上海交通大学机械系统与振动国家重点实验室 上海 200240; \\ 2. 清华大学摩擦学国家重点实验室 北京 100084)
}

\begin{abstract}
摘要: 表面作为零部件的 “指纹”, 其科学性是开展后续润滑、接触、摩擦磨损、监测控制等研究的基础。现有学者多从单 层表面视角出发理解表面, 而忽视了双工序、磨损等表面存在的双高斯分层特征。少量学者受到双工序表面加工制造过程的 启发, 提出了双高斯分层表面理论, 并成功拓展至磨损表面的研究。从表面表征仿真、润滑接触、摩擦磨损、监测控制四个 方面归纳总结了国内外有关双高斯分层表面理论的研究工作, 特别关注该理论在机械密封领域的应用, 进而提出了后续可深 入探讨的双高斯研究方向, 以期为机械密封的设计与研究提供理论和工程指导。
\end{abstract}

关键词: 机械密封; 表面形貌; 润滑; 接触; 摩擦磨损; 声发射

中图分类号: TH136

\section{Review on Mechanical Seals Using a Bi-Gaussian Stratified Surface Theory}

\section{HU Songtao $^{1}$ HUANG Weifeng ${ }^{2} \quad$ SHI Xi $^{1} \quad$ PENG Zhike $^{1} \quad$ LIU Xiangfeng ${ }^{2}$}

(1. State Key Laboratory of Mechanical System and Vibration, Shanghai Jiao Tong University, Shanghai 200240;

2. State Key Laboratory of Tribology, Tsinghua University, Beijing 100084)

\begin{abstract}
Surface, serving as the fingerprint of a component, is the basis of the following investigations on lubrication, asperity contact, wear and friction, monitoring and controlling, etc. Researchers usually understand a surface from the perspective of a classical single-stratum surface, while ignore the bi-Gaussian stratified characteristic exists in a two-process or worn surface. Some researchers proposed a bi-Gaussian stratified surface theory from the processing and manufacturing of two-process surfaces, and then extended to worn surfaces. In the present study, a review of bi-Gaussian-stratified-surface-theory works is given with respect to surface characterization and simulation, lubrication and contact, wear and friction, and monitoring and controlling, which particularly focuses on the field of mechanical seals. The interesting bi-Gaussian issues those can be further explored are also discussed. It will benefit the design and analysis of mechanical seals.
\end{abstract}

Key words: mechanical seal; surface topography; lubrication; asperity contact; wear and friction; acoustic emission

\section{0 前言}

机械密封, 通常作为轴端密封而使用, 其依靠 弹性元件对密封副的预紧和介质压力与弹性元件压 力的压紧来实现密封 ${ }^{[1-3]}$ 。对于接触式机械密封, 其 在正常运行工况下长期处于混合润滑状态, 密封副 端面之间存在粗粘峰接触; 对于非接触式机械密封, 其在正常运行工况下处于流体润滑状态, 但在主机 启停、盘车、暖机等过程或者主机存在振动、冲击 等扰变工况下，其密封副端面依然会出现粗㮞峰接 触问题。密封副端面的接触会引起不同程度的摩擦

* 国家自然科学基金(11632011, 11372183)、国家科技支撑计划 (2015BAA08B02)和中国博士后科学基金(2017M621458)资助项目。 20180224 收到初稿, 20180711 收到修改稿
磨损, 轻则造成密封性能劣化, 重则诱发密封突发 性失效导致主机连锁停机甚至安全事故。因此, 在 设计和研究机械密封时, 有必要就其摩擦磨损机理 展开深入的探讨, 以保证长使役周期内密封性能的 稳定与可靠。

根据美国材料与试验协会关于磨损的定义(即, 相互接触物体表面之间相对运动而造成的物体表面 损伤), 需要以表面、接触与动力学为基础开展摩擦 磨损研究。此外, 较为完整的研究体系尚需涵括在 线监测与控制环节。

(1) 表面研究。

在表面研究方面, HU 等 ${ }^{[4-9]}$ 认为表面可以视为 零部件的 “指纹” ：可用于评价、指导加工制造; 可用于展现、改善性能功能; 可用于探索、揭示机 理本质; 若配合以声发射这一 “指纹识别器” , 则 
可用于实现在线监控功能。表面研究主要包括几何 形貌(粗粘度与波纹度)、织构、材料、涂层等领域, 本文仅关注粗粘度尺度的表面形貌研究。表面形貌 研究可划分为表征和仿真两大部分。

表面表征, 通过分析表面数据提炼出可反映目 标表面某些特性的特征参数。在初期, 学者采用表 面高度的算数平均偏差 $R_{\mathrm{a}}$ 来表征二维轮廓数据。随 着测量技术与数学理论的发展, 二维表面参数不断 增加直至在 20 世纪 70 年代出现 “参数大爆炸” 。 在三维测量技术诞生后, 为避免重演二维表面参数 的覆辙, 以 Birmingham 大学学者为主的欧洲三维分 析工作委员会于 1994 年前后提出了基于 14 个三维 表面参数的表征集, 包括幅值、空间、混合和功能 四大类。这其中 ${ }^{[10-11]}, S a$ 和中心矩参数族 $(S q, S s k$ 和 $S k u$ )为常用表面参数。不同于 $S a$ 多被应用于工程 实际, 均方根偏差 $S q$ 更倾向于理论研究, 两者均可 表征表面形貌相关于其均值面的波动程度。三阶矩 偏态 Ssk 用于表征表面形貌的不对称性, 四阶矩峰 态 $S k u$ 用于表征表面形貌的分散集中程度。除了上 述幅值表面参数外, 常用的表面参数还多包括自相 关函数、粗粘峰密度、纵横比、纹理方向、最快下 降自相关长度等空间表面参数。基于表面表征的结 果, 学者发现, 工程中大量表面的形貌高度的概率 密度函数满足高斯分布, 其 $S s k$ 为 $0, S k u$ 为 3 。然 而, 工程中同时也存在大量表面体现出非高斯分布 特征 ${ }^{[12-14]}$; 且高斯表面在经历摩擦磨损后也往往呈 现为非高斯分布 ${ }^{[15-22]}$ 。非高斯表面的 Ssk 不为 0 ,

$S k u$ 不为 3 。

表面形貌数据可以通过试验和数值仿真两种方 式来获取。由于后者更为经济、稳定、灵活和高效, 成为学者研究的重点。当前, 表面仿真方法大体可 划分为时间序列模型 ${ }^{[23-28]}$ 和函数序列模型 ${ }^{[29-31]}$ 两大 类, 前者又可进一步细分为自动回归模型、移动平 均模型和混合模型。自动回归模型存在高阶局限性, 仅原点附近的自相关函数能被较高程度地仿真还 原 ${ }^{[25]}$ 。此外, 学者还提出了随机中点位移模型 ${ }^{[32]}$ 、 神经网格模型 ${ }^{[33]}$ 和 Monte Carlo 模型 ${ }^{[34]}$ 。采用上述 模型, 以尺寸、节点数、Sq、自相关函数类型和相 关长度为输入, 可以仿真高斯表面。在具体求解模

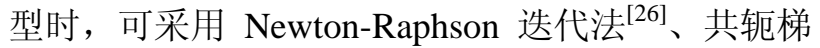
度法 ${ }^{[27]}$ 等直接法和快速傅里叶变换法 ${ }^{[0-31]}$; 后者的 计算效率更高, 存储空间更小。非高斯仿真方法则 以高斯仿真为基础, 采用 Johnson 转换系统 ${ }^{[35-37]}$ 用 非高斯序列代替高斯序列, 以生成具有指定偏、峰 态的目标非高斯表面。

目前, 大量学者对机械密封端面开展了表征分
析, 关注 $S q 、 S s k$ 、粗粮峰密度等表面参数 ${ }^{[15-19,38-47]}$ 。 进一步, 采用 Johnson 非高斯仿真方法重构了三种 不同材料配副的机械密封端面 ${ }^{[19]}$, 如图 1 所示。

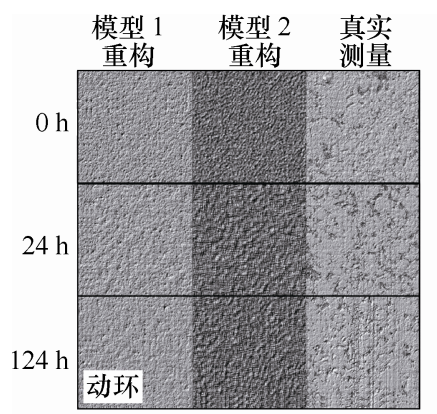

(a) 动环

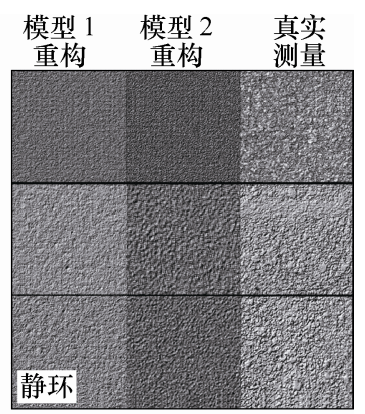

(b) 静环
图 1 磨损的机械密封端面与其 Johnson 非高斯仿真结果 ${ }^{[19]}$

(2) 接触研究。

在接触研究方面, 早期的接触模型以单个粗䊁 峰为研究对象, 先后建立了 Hertz 弹性接触模型 ${ }^{[48]}$ 和 Bowden 塑性变形模型 ${ }^{[49]}$ 。在 1966 年, GREENWOOD 等 ${ }^{[50]}$ 基于统计学理论建立了粗楉表 面间的弹性接触模型。随后, PULLEN 等 ${ }^{[51]}$ 基于体 积守恒证明了由 ABBOTT 和 FIRESTONE ${ }^{[52]}$ 提出的 塑性接触模型。CHANG 等 ${ }^{[53]}$ 基于体积守恒, 提出 了弹塑性接触模型。伴随着测量技术和数学理论的 发展, 学者采用斜率分布、曲率分布、功率谱密度 和自相关函数等表面参数发展统计学接触模型 ${ }^{[54-57]}$ 。 然而, MAJUMDAR 等 ${ }^{\left[{ }^{[8]}\right]}$ 指出上述统计学表面参数 受仪器分辨率的影响, 原因为工程表面具有分形特 性 ${ }^{[59]}$ 。为此, MAJUMDAR 等 ${ }^{[60]}$ 基于 WeierstrassMandelbrot 函数提出了粗粘表面间的分形统计学接 触模型; YAN 等 ${ }^{[61]}$ 进一步将分形接触模型拓展至三 维形貌。陆续有学者对分形接触模型展开深入的分 析 $^{[62-63]}$ 。事实上, 在分形统计学模型中, 随着载荷 与真实接触面积增大, 粗鋉峰出现由塑性接触向弹 性接触转化的现象, 违背了经典统计学接触模型结

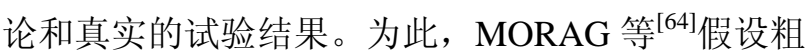
粘峰实际的形变量区别于干涉量, 建立了修正的混 合统计学模型。除了统计学接触模型, 学者亦开展 确定性接触模型研究 ${ }^{[65-81]}$ 。但在工程实际中, 表面 形貌复杂多变, 制约了确定性模型的应用。

目前, 针对接触式机械密封, 粗粘峰接触模型 是其混合润滑建模过程中不可规避的 ${ }^{[22-89]}$ 。而针对 非接触式机械密封, 先后有学者将经典统计学、分 形统计学接触模型应用于动力学特性研究 ${ }^{[90-94]}$, 如 图 2 所示。

(3) 动力学研究。

在动力学研究方面, 机械密封的动力学特性主 要包括正常运行阶段的稳态特性、启停阶段的瞬态 


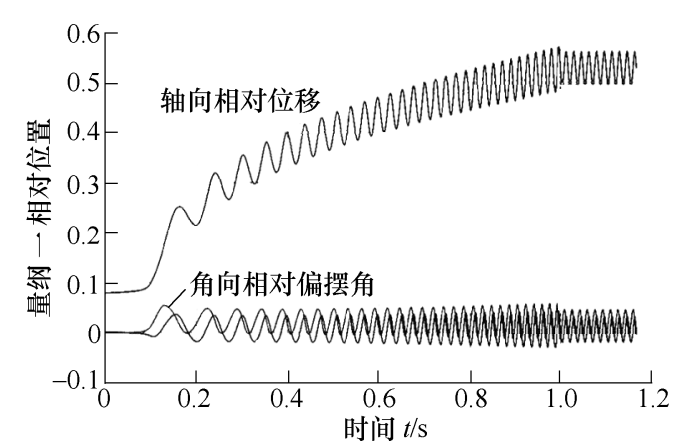

图 2 干气密封启动阶段的动力学特性 ${ }^{[92]}$

特性、以及受扰运行阶段的瞬态特性。学者将动、 静环简化为两个薄圆盘, 建立综合考虑轴向和角向 行为的动力学简化物理模型, 如图 3 所示。采用全 数值法进行模型的求解, 可得到膜压分布和密封环 运动状态的完整历程 ${ }^{[95-98]}$ 。为了提升计算效率, 采 用解析 ${ }^{[82]}$ 、半解析 (摄动 ${ }^{[99-107]}$ 和阶跃 ${ }^{[108-115]}$ ) 法和本 征正交分解法 ${ }^{[116-117]}$ 在各自限制条件下开展模型简 化工作。随后, 粗粘峰接触模型被耦合到上述动力 学模型。

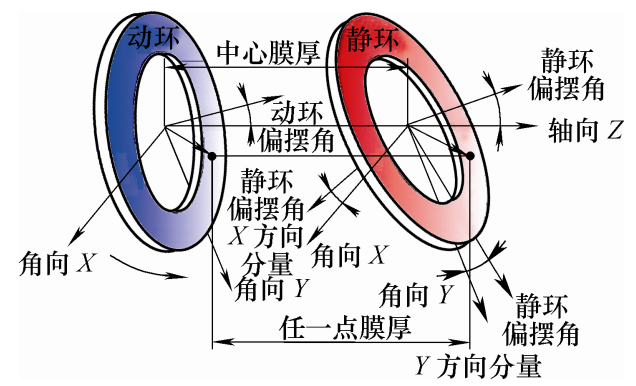

图 3 机械密封的动力学简化建模

(4) 摩擦磨损研究。

在摩擦磨损研究方面, 针对机械密封, 滑动摩 擦磨损类型为主要研究对象。考虑到表面的 “指纹” 角色, 大量学者希望建立起表面形貌与摩擦磨损特 性之间的关系。事实上，摩擦磨损特性内容宽泛， 涵括了耐磨性 ${ }^{[20-22,118-124]}$ 、摩擦因数 ${ }^{[125-136]}$ 、转换 层 ${ }^{[126-127,129,131]}$ 等, 本文仅关注耐磨性和摩擦因数这 两个热门研究领域。

局部磨损量不同于磨损重量, 它从表面形貌角 度评价耐磨性。如图 4a 所示, 一个表面在磨损前后 的两条材料比曲线可以依据某一个基准高度进行平 移, 而平移后的两条材料比曲线与表面高度坐标轴 所围成的面积即被定义为局部磨损量 ${ }^{[118-121]}$ 。除了 面积差, KUMAR 等 ${ }^{[119]}$ 通过线性拟合得到拟合直线 在表面高度坐标轴的投影值, 定义为磨损深度, 如 图 4b 所示。无论是面积差型还是高度差型的局部磨 损量, 都需要确保磨损前后表面测量的定位精度。 当然, 也有学者仅通过磨损后的表面形貌来获取局 部磨损量 ${ }^{[122-123,137]}$ 。除了上述的轮廓测定法, 有学
者 ${ }^{[20-22,121,137]}$ 希望搜寻出个别的表面参数或组合, 以 直接评价局部磨损量。与耐磨性研究类似地, 学者 尝试建立起摩擦因数或扭矩与表面形貌之间的关

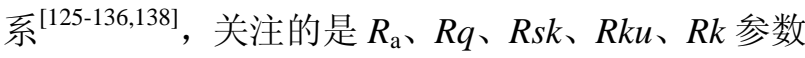
族、以及 $R t-R p-R v$ 磨损评价指标等表面参数。除了 上述摩擦磨损机理试验研究, 学者也陆续开展了密 封台架试验 ${ }^{[15-19,38-40]}$ 。

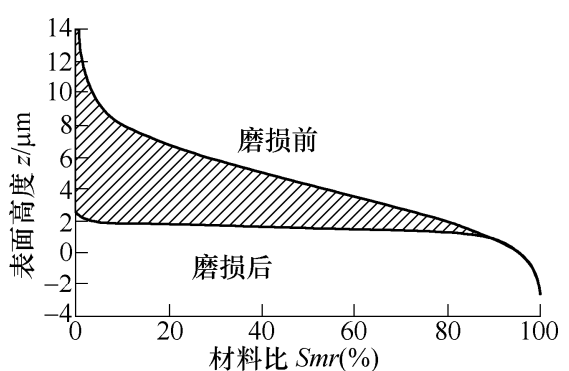

(a) 面积差型

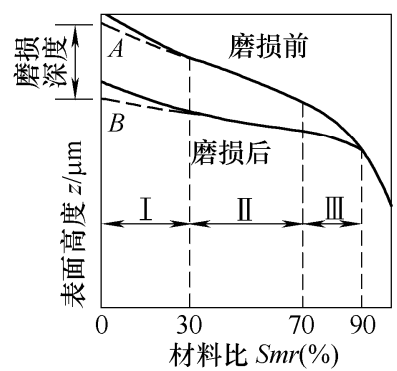

(b) 高度差型

图 4 面积差型 ${ }^{[121]}$ 和高度差型 ${ }^{[119]}$ 局部磨损量

(5) 监测控制研究。

在监测控制研究方面, 学者以膜厚、温度、扭 矩等物理量为监测指标，以气体加压、机电制动、 压电制动、电磁制动等方式控制密封 ${ }^{[139-151]}$ 。其中, 声发射技术作为一种直接的端面接触监测手段, 被 从车刀、齿轮、轴承等领域 ${ }^{[152-160]}$ 引入到机械密封 领域 ${ }^{[161-170]}$ 。图 5 为 HUANG 等 ${ }^{[166-167]}$ 使用声发射技 术监测干气密封在启停和受扰运行阶段的端面接触 状态。

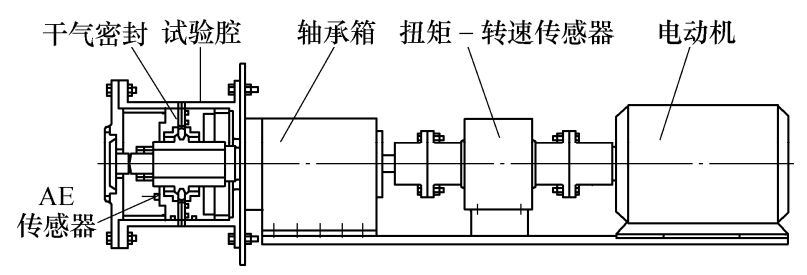

图 5 声发射技术监测干气密封端面接触 ${ }^{[166,167]}$

(6) 小结。

综上所述，机械密封的摩擦磨损研究涉及到表 面、润滑、接触、动力学、摩擦学、测控等多学科 交叉。这其中, 表面作为密封副的“指纹”, 其科学 性是开展后续其他领域研究的基础。如上所述, 在 工程实际中，机械密封端面在经历摩擦磨损前可呈 
现为高斯或非高斯分布, 在经历摩擦磨损后呈现为 非高斯分布。从现有研究可知, 绝大多数学者从单 层表面角度出发认识表面, 进而开展润滑、接触、 动力学、摩擦磨损以及监测控制研究。

事实上，在非高斯表面中，存在一类双高斯表 面, 主要包括双工序(如平顶街磨)表面和磨损表面。 双高斯表面不仅具备非高斯特征, 更要求学者从分 层表面的角度来重新认识表面本质。机械密封端面 在经历摩擦磨损后属于双高斯表面范畴, 在经历摩 擦磨损前由于先后历经多道加工工序(与双工序表 面类似), 也有可能呈现出双高斯分层特征。本文将 从表面表征仿真、润滑接触、摩擦磨损、监测控制 这四个方面归纳整理目前国内外双高斯分层表面理 论的研究现状, 特别是涉及机械密封的相关研究, 希望为机械密封的设计与研究提供一份系统的背景 资料。

\section{1 双高斯分层表面的表征与仿真}

\section{1 表面表征}

对于非高斯表面, 学者已通过采用 $S s k$ 和 $S k u$ 来开展表征分析, 但仍忽视了其可能存在的分层特 征。THOMAS ${ }^{[171]}$ 采用如图 6a 所示模型来模拟磨损 表面: 高斯表面被某一平行于其均值面的光滑平面 截为上下两部分, 下半部分与光滑平面共同构成了 磨损表面。事实上, 对于实际的磨损表面, 其截取 面并非完全光滑, 其表面高度同样服从高斯分布, 只是尺度远小于被截的高斯表面 ${ }^{[172]}$, 如图 $6 \mathrm{~b}$ 所示。 上述由两个高斯分量构成的表面被称为双高斯分层 表面 ${ }^{[4-6,8-9,17,173-174]}$ 。双高斯表面思想源自汽缸表面 的平顶行磨这一双工序加工手段 ${ }^{[12-14]}$; 随后, 鉴于 摩擦磨损过程与双工序加工制造过程的相似性, 被

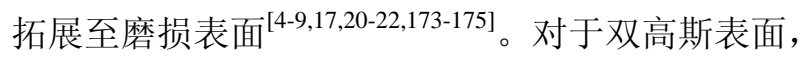
其上高斯分量用于承受载荷与抵抗磨损, 而下高斯 分量用于容纳碎屑与润滑介质。

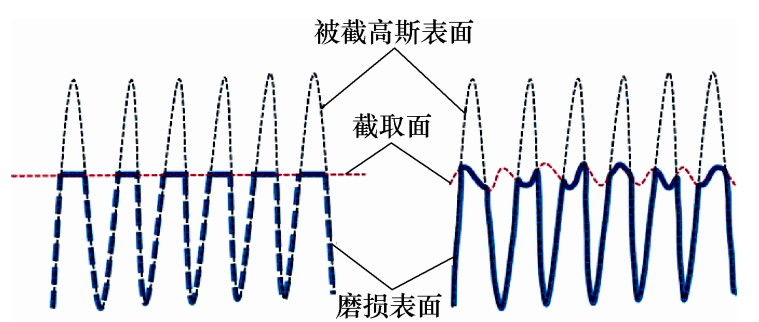

(a)

(b)

图 6 双高斯分层表面的解释

为了表征双高斯表面, 学者提出了概率材料比 曲线方法。材料比曲线又称为支承面曲线、Abbott
曲线, 可由表面形貌数据计算得到, 其横坐标(概率 坐标)为材料比, 纵坐标(高度坐标)为表面高度, 其 物理意义为当某一高度的水平面截取粗粘表面时, 所截面积占粗鋉表面的百分比。概率材料比曲线由 材料比曲线的概率坐标经非等比例压缩至标准偏差 坐标而形成。经过压缩后, 标准偏差坐标中的 $-3,-2$, $-1,0,1,2$ 和 3 分别对应于概率坐标中的 $\quad 0.13 \%$, $2.28 \%, 15.87 \%, 50 \%, 84.13 \%, 97.72 \%$ 和 $99.87 \%$ 。 WILLIAMSON ${ }^{[176]}$ 发现高斯表面的概率材料比曲线 呈线性, 斜率为 $S q$, 截距为均值面。由此可知, 双 高斯表面的概率材料比曲线必然体现为双线性。据 此, 学者基于分段线性回归思想, 先后提出了简单 间断分离方法 ${ }^{[14]}$ 和 ISO 间断分离方法 ${ }^{[177]}$, 以得到 上高斯分量的均方根偏差 Spq、上高斯分量的均值 面 $z_{\mathrm{mu}}$ 、下高斯分量的均方根偏差 $S v q$ 、下高斯分量 的均值面 $z_{\mathrm{ml}}$ 、过渡点的材料比 $S m q$ 及对应的高度 $z_{\mathrm{k}}$ 。与简单间断分离方法不同, ISO 间断分离方法 先通过复杂算法排除概率材料比曲线上存在的非线 性区域(即, 边远峰区、边远谷区以及过渡区), 再 开展分段线性回归。

然而, $\mathrm{HU}$ 等 ${ }^{[4]}$ 指出, 间断分离方法主观地认为 概率材料比曲线是由两条直线段在某一拐点处简单 拼接而成, 违背了概率密度函数需要满足单位积分 面积的要求。进一步, 如图 7 所示 ${ }^{[4]}$, 通过提出表 面混合理论, 给出了由两个初始表面 $\left(S_{1} 、 S_{2}\right)$ 的概率 密度函数 $\left(f_{1} 、 f_{2}\right)$ 和累积分布函数 $\left(P_{1} 、 P_{2}\right)$ 构建混合表 面 $S_{3}$ 的概率材料比曲线的连续型解析式, 实现了概 率材料比曲线的整体拟合, 即建立了连续分离方法。 图 8 为 ISO 间断分离方法与连续分离方法的比较示 意图 ${ }^{[8]}$ 。进一步, 通过对比研究, $\mathrm{HU}$ 等 ${ }^{[5-6,173]}$ 发现

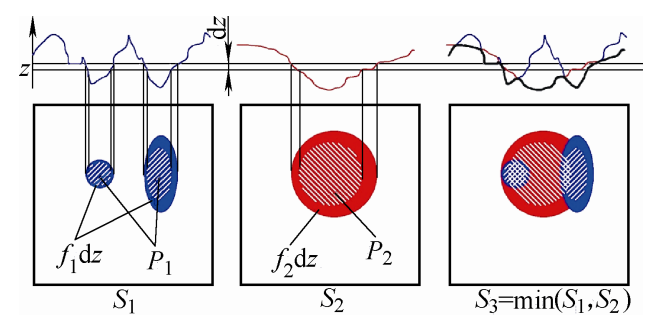

图 7 表面混合理论

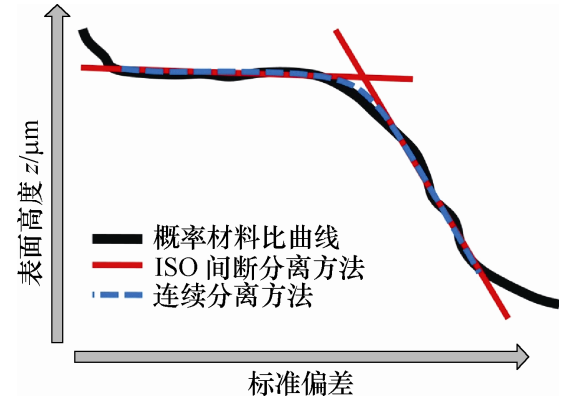

图 8 ISO 间断分离方法与连续分离方法的比较 
连续分离方法不仅能满足概率密度函数单位积分面 积的物理要求, 同时效率提升了约 100 倍, 有效解 决了工程实际中存在的概率材料比曲线波动以及分 量表面粗粘度尺度相关性问题, 但是其无法自行识 别合理的应用区间。

首先，上述间断分离方法和连续分离方法都基 于概率材料比曲线的拟合。其次, 在工程实际中, 双高斯表面的上高斯分量用于承受载荷与抵抗磨 损; 若上高斯分量表征存在较大误差, 误差将引入 后续的接触、摩擦磨损等机理研究中; 由此可见上 高斯分量的重要性。综合上述两点考虑, HU 等 ${ }^{[5]}$ 提出以偏态为收玫指标, 直接从目标表面中截取出 上高斯分量, 以保真全部的上高斯分量信息, 命为 截取分离方法。但在实际分析过程中发现, 截取分 离方法需要在下高斯分量表征环节沿用传统的概率 材料比曲线拟合方式, 且不适合上高斯分量比重较 小的场合。

综上, 连续分离方法比间断分离方法和截取分 离方法更适合于双高斯表面的表征分析。

\section{2 表面仿真}

与 Johnson 非高斯仿真方法一样, 双高斯仿真 方法也以高斯表面的仿真为基础。PAWLUS ${ }^{[178]}$ 基于 叠加原理思想提出了双高斯仿真方法, 其基本流程 如图 9 所示 ${ }^{[6]}$ : (1) 独立生成上下高斯分量; (2) 输 入分量均值面的间距; (3) 在各节点处保留数值较小 的表面高度值, 形成双高斯表面, 并对结果表面进 行均值面归零化处理。HU 等 ${ }^{[4-6,173]}$ 对真实磨损表 面、基于连续分离方法的 Pawlus 双高斯重构表面、 基于简单间断分离方法的 Pawlus 双高斯重构表面、 基于 ISO 间断分离方法的 Pawlus 双高斯重构表面和 Johnson 非高斯重构表面进行了比较分析, 如图 10 所示 ${ }^{[173]}$ 。他们发现, Pawlus 双高斯仿真方法更接 近真实表面磨损情况: (1) 能生成分层表面, 突破 Johnson 非高斯仿真方法只能生成单层表面的局限; (2)能克服 Johnson 非高斯仿真方法无法兼顾偏态和 峰态的不足。然而, 他们同时指出, Pawlus 双高斯 仿真方法由于分量节点丢失, 在分量相关长度识别

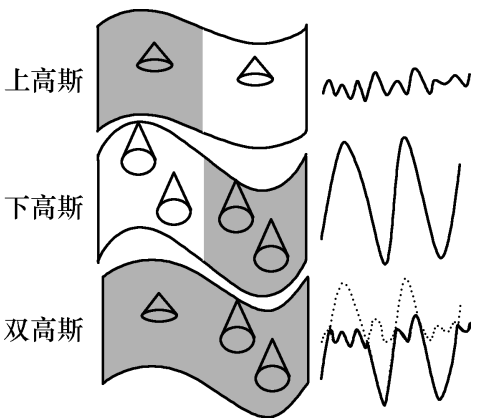

图 9 Pawlus 双高斯仿真方法流程
方面存在不足。进而, 他们采用迭代算法进行了方 法改进，并证明改进后的 Pawlus 双高斯仿真方法所 产生的自相关函数比 Johnson 非高斯仿真方法更为 灵活, 如图 11 所示 ${ }^{[6]}$ 。

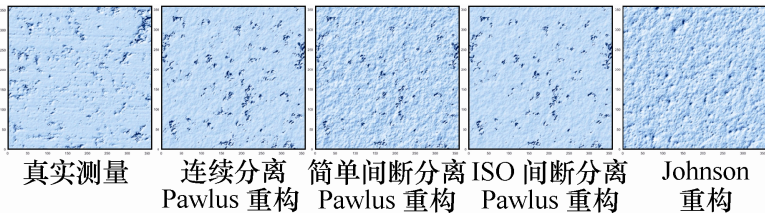

图 10 可视化的表面仿真结果

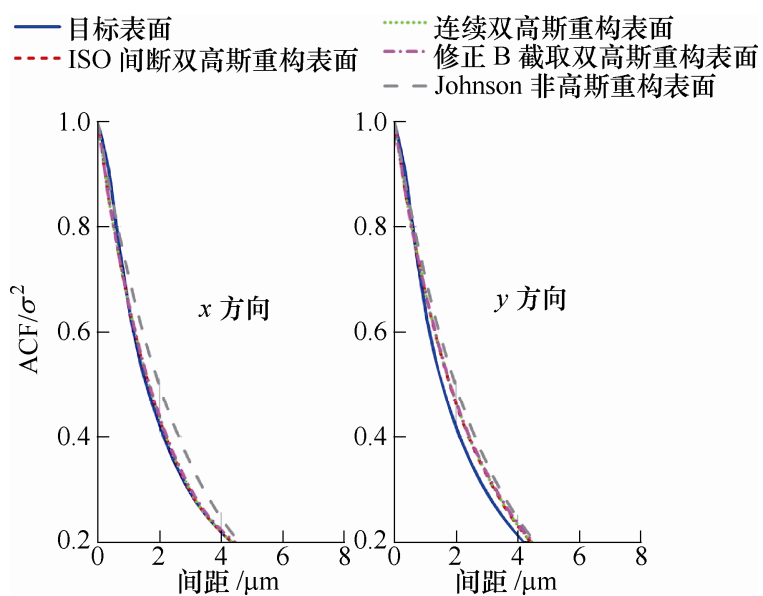

图 11 表面仿真结果的自相关函数

综上，与 Johnson 非高斯仿真方法相比，迭代 修正后的 Pawlus 双高斯仿真方法在重构实际磨损 表面时具有表面分层、偏态与峰态兼顾、自相关函 数灵活等优点, 突破了单层表面的局限, 更为接近 真实表面磨损情况。

\section{3 在机械密封的应用}

在表面表征方面，概率材料比曲线方法已被应 用于双工序表面的制造加工 ${ }^{[12-14]}$ 、润滑与接触性能 研究 ${ }^{[17,173-174]}$ 、以及摩擦学特性研究 ${ }^{[8-9,20-22]}$ 。这其中, 仅有 LEEFE 等 ${ }^{[17]}$ 的研究涉及到密封领域。LEEFE 率先尝试将双高斯分层表面理论应用于机械密封, 采用间断分离方法表征磨损表面的二维轮廓。而 $\mathrm{HU}$ 等 ${ }^{[4-6,8-9,173-174]}$ 则对方法本身开展深入的探究, 进 而向润滑、接触、摩擦磨损等科学问题推广。而在 表面重构方面，PAWLUS ${ }^{[178]}$ 和 HU 等 ${ }^{[4-6,173]}$ 采用了 双高斯仿真方法。这其中, 仅 $\mathrm{HU}$ 等 ${ }^{[4-6,172]}$ 的工作属 于密封领域。

\section{2 双高斯分层表面的润滑与接触}

\section{1 表面润滑}

$\mathrm{HU}$ 等 ${ }^{[173]}$ 采用有限差分法和松弛迭代法建立了 确定性模型, 围绕干气密封与水膜机械密封, 研究 了表面形貌对于气膜润滑和含空化效应水膜润滑性 
能的影响。图 12 为构建的研究单元体 ${ }^{[173]}$, 等效粗 糙面在 $x$ 方向上相对于光滑面以 $U$ 的速度平移。对 照实际的机械密封环境, 上述边长为 $L_{\mathrm{x}}$ 的研究单元 体在 $y$ 方向上存在 $p_{2}-p_{1}$ 的压差, 在 $x$ 方向上为周期 性边界条件, 等效粗粘面的均值面与光滑面的间距 为 $h_{0}$ 。由于所研究的表面为非周期表面, 强行施加 周期性边界条件会引入额外的边界效应, 降低结果 的可信度, 故在实际研究中将周期性边界条件改为 狄利克雷边界条件, 即研究对象已由密封转化为轴 承。如图 13 所示 ${ }^{[173]}$, 他们发现, 当膜厚与粗粘度 处于同一尺度时, 表面形貌对润滑性能的影响极大。 非高斯特征易产生极值节点, 造成流体压力尖峰, 出现较大空化区, 产生较大流体承载力。而双高斯 分层特征比非高斯特征更接近真实表面磨损情况。

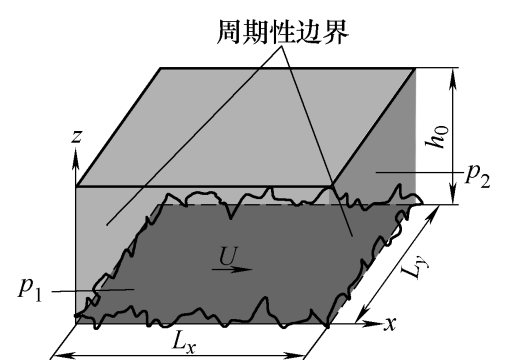

图 12 利用确定性模型研究润滑性能的单元
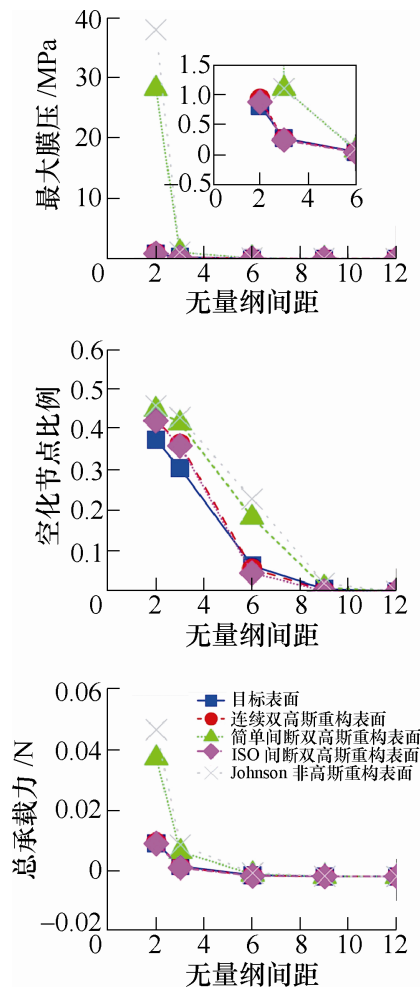

图 13 利用确定性模型研究润滑性能

\section{2 表面接触}

$\mathrm{LEEFE}^{[17]}$ 在采用间断分离方法表征磨损表面 的二维轮廓后, 基于 Greenwood-Williamson 模型 ${ }^{[50]}$ 建立了间断双高斯分层粗粘峰统计学接触模型。过
程中, 其从二维轮廓参数估算三维粗粘峰参数, 用 于所建的分层模型。

$\mathrm{HU}$ 等 ${ }^{[173]}$ 首先采用确定性模型研究表面形貌对

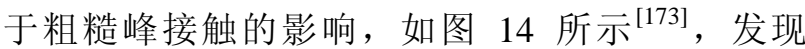
Johnson 非高斯特征由于具有极高的表面节点, 当间 距减小时率先出现粗龯峰的接触，且在相同间距下 体现出较大的平均接触压力和真实接触面积比例。 双高斯分层特征比非高斯特征更接近真实表面磨损 情况。随后, HU 等 ${ }^{[174]}$ 修正了 LEEFE 建模过程中 的部分假设，建立了修正的间断双高斯分层粗䊁峰 统计学接触模型，如图 15 所示 ${ }^{[174]}$ 。模型中，存在 两个变量系列: 在表面高度系列中, $\sigma_{\mathrm{u}}$ 和 $\sigma_{\mathrm{I}}$ 即为第 1.1 节表面表征中的 $S p q$ 和 $S v q$; 含下标 $\mathrm{s}$ 的变量系 列由将概率材料比曲线方法应用于粗糙峰高度的概 率材料比曲线所得, 是表面高度系列变量在粗䊁峰 高度的拓展。进一步, $\mathrm{HU}$ 等 ${ }^{[174]}$ 采用连续分离方法 中构建的连续概率密度函数, 建立了连续双高斯分 层粗䊁峰统计学接触模型; 利用确定性接触模型验 证了上述分层统计学接触模型的准确性, 如图 16 所示 ${ }^{[174]}$ 。
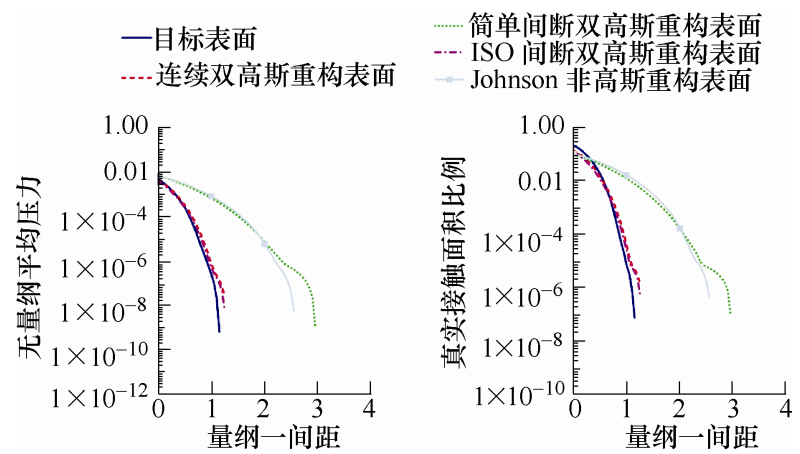

图 14 利用确定性模型研究接触性能

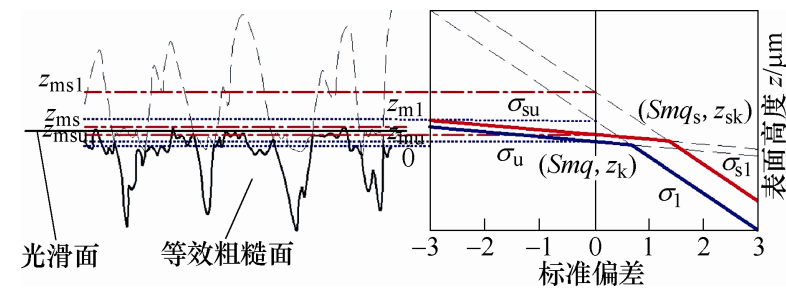

图 15 修正的间断双高斯分层粗䊁峰统计学接触模型

PAWLUS 团队 ${ }^{[179-180]}$ 则基于双高斯分层表面理 论重新定义了接触机理研究中的关键参数(即塑性 系数), 并进行了试验分析。而该修正塑性系数被 $\mathrm{HU}$ 等 ${ }^{[8]}$ 应用于摩擦磨损机理试验的分析, 用于判断 弹塑性范畴。

\section{3 在机械密封的应用}

PAWLUS 团队 ${ }^{[179,180]}$ 将双高斯分层表面理论应 用于表面接触问题, 关注的是塑性系数的重新定义。 在密封领域，LEEFE ${ }^{[17]}$ 采用间断分离方法表征磨损 
表面的二维轮廓后, 估算三维粗粘峰参数, 应用于 其初步建立的间断双高斯分层粗粘峰统计学接触模 型。而 HU 等则首先采用确定性模型研究表面形貌 对于气膜润滑、含空化效应水膜润滑与粗粘峰接触
的影响 ${ }^{[173]}$; 进一步，修正了 LEEFE 间断双高斯分 层粗粘峰统计学接触模型, 同时建立了连续双高斯 分层粗粘峰统计学接触模型, 利用确定性接触模型 验证了上述两个分层统计学接触模型的准确性 ${ }^{[174]}$ 。
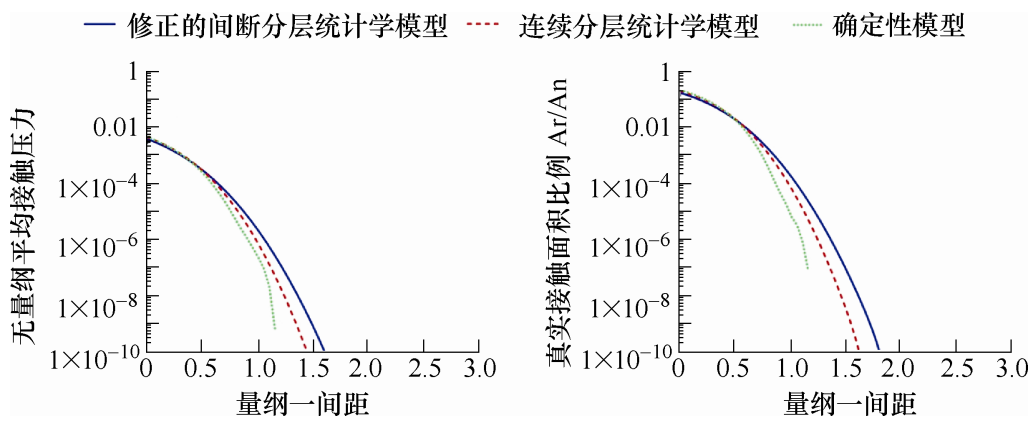

图 16 双高斯分层粗粘峰统计学接触模型的验证

\section{3 双高斯分层表面的摩擦磨损}

\section{1 机理试验}

目前, 已有学者开始尝试从双高斯分层表面角度 出发, 研究局部磨损量 ${ }^{[8-9,20-22,121]}$ 和摩擦因数 ${ }^{[8-9,90-22]}$ 。 针对图 4a 面积差型局部磨损量, Corral 等 ${ }^{[121]}$ 提出 将材料比曲线替换为概率材料比曲线, 如图 17 所 示。进一步, $\mathrm{HU}$ 等 ${ }^{[8-9]}$ 将高度差型局部磨损量拓展 至概率材料比曲线。这其中, 他们借鉴材料比曲线 拟合 30\%至 $70 \%$ 区域内数据点, 将概率材料比曲线 的拟合区间设置为 -2 至 2 。

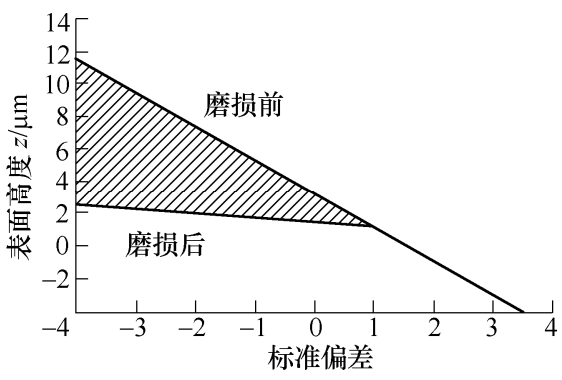

图 17 面积差型局部磨损量关于概率材料比曲线的修正 ${ }^{[121]}$

PAWLUS 才队 ${ }^{[20-22]}$ 也陆续开展了局部磨损量和 摩擦因数的研究。这其中, GRABON 等 ${ }^{[20]}$ 研究了 灰口铸铁和涂铬 45 钢材料配副的往复滑动摩擦。 PAWLUS 等 ${ }^{[21]}$ 利用四球试验台, 研究了 100CR6 钢 和 42CRMO4 钢材料配副的干滑动摩擦。而 DZIERWA 等 ${ }^{[22]}$ 则利用球盘试验台, 开展 $100 \mathrm{Cr} 6$ 钢 和 $42 \mathrm{CrMo} 4$ 钢材料配副在干摩擦和乏油润滑条件 下的研究。

$\mathrm{HU}$ 等 $^{[8-9]}$ 则关注于时间效应, 设计了两套不同 的试验方案, 利用标准旋转试验台, 研究了密封用 碳化硅与碳石墨材料配副在干滑动过程中表面形貌 的演化历程。从两套试验方案的结果中, 他们建立
起软硬材料的双高斯表面参数演化模型, 如图 18 所示 ${ }^{[9]}$ 。上述模型从表面形貌的角度解释了摩擦磨 损过程中跑合与稳定阶段的成因，特别是回答了 “在稳定阶段, 表面形貌依然在不断变化, 但摩擦 因数可以保持稳定” 的疑问。

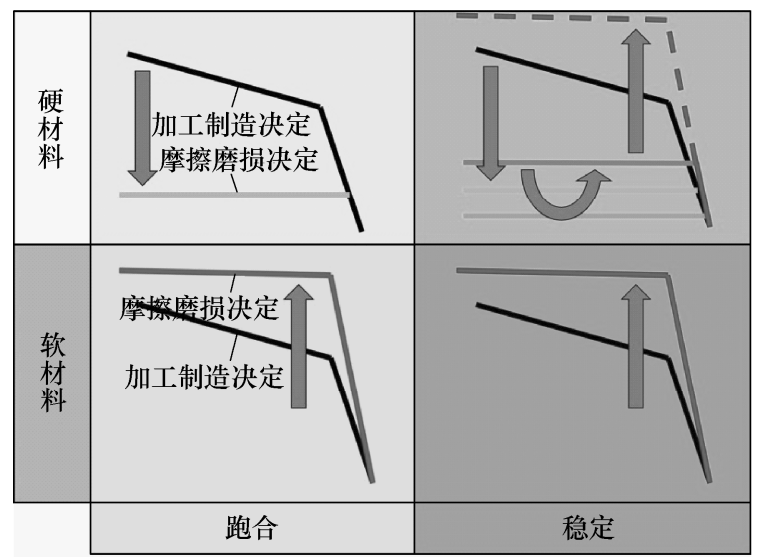

图 18 双高斯表面参数演化模型

\section{2 密封台架试验}

PAWLUS 团队 ${ }^{[20-22]}$ 的机理试验, 针对的是齿轮、 缸机, 而 HU 等 ${ }^{[8-9]}$ 则围绕机械密封开展研究。目前, $\mathrm{HU}$ 等已进一步尝试将上述机理试验 ${ }^{[8-9]}$ 的研究方案 和试验经验应用于图 5 所示的密封台架。试验结果 表明: (1) 台架试验中密封端面表面参数的演化历程 与标准摩擦磨损试验所得结论一致, 实现了摩擦磨 损机理研究在实际机械密封中的应用; (2) 双高斯表 面参数演化历程成功识别出了端面磨合、粘着磨损 以及深度划痕过程或现象; (3) 机理试验中所得的两 个双高斯表面参数演化模型为统一体。

\section{4 双高斯分层表面的监测控制}

$\mathrm{HU}$ 等 ${ }^{[4-9]}$ 提出表面可视为零部件的 “指纹” , 
则必然涉及到“指纹”的识别问题。在工程实际中, 目前尚未见有设备能够实时地获得零部件表面形貌 的完整数据, 这限制了表面 “指纹” 这一功能在监 控方面的应用。为此, $\mathrm{HU}$ 等基于课题组之前的研 究 ${ }^{[166-167]}$, 将声发射技术视为一种 “指纹识别器”, 应用于密封台架试验 ${ }^{[181]}$ 。过程中, 他们实时测量了 机械密封的泄漏率、扭矩和声发射信号, 对照表面 形貌的演化历程, 发现两者存在较高的关联性。这 其中, 泄漏率的演化规律可以由双高斯表面参数的 演化历程来解释; 声发射信号可以识别出端面磨合 阶段, 且其演化规律可由粗糙峰参数的演化历程来 解释。通过密封台架试验, 初步验证了声发射作为 “指纹识别器” 的可行性。随后, $\mathrm{HU}$ 等 ${ }^{[182]}$ 已开始 尝试基于双高斯分层表面理论建立声发射模型, 开 展机理研究。

\section{5 结论与展望}

总结了国内外有关双高斯分层表面理论的研究 工作，特别关注该理论在机械密封领域的应用。所 取得的主要成果可概括为以下几点。

(1) 学者受双工序表面加工制造过程的启发, 提出了双高斯分层表面理论, 并拓展至磨损表面。 该理论被应用于机械密封领域。

(2) 表面表征方面: 先后提出了简单间断分离、 ISO 间断分离、连续分离和截取分离方法。综合考 虑, 连续分离方法最适合于双高斯表面的表征。上 述表征方法被应用于机械密封领域。

（3）表面仿真方面: 基于叠加原理提出了双高 斯仿真方法, 并利用迭代算法识别分量相关长度。 上述仿真方法被应用于机械密封领域。

(4) 润滑与接触方面: 利用确定性模型研究了 表面形貌对气膜润滑、含空化效应水膜润滑以及粗 粘峰接触的影响。进一步, 先后建立了间断、修正 间断和连续双高斯分层粗糙峰统计学接触模型。上 述模型被应用于机械密封的数值分析, 缺乏试验研 究。

(5) 摩擦磨损方面: 尝试建立起表面形貌与耐 磨性、摩擦因数等摩擦学特性间的关联性。建立了 双高斯表面参数演化模型, 从表面形貌角度解释了 跑合与稳定阶段的成因, 特别是回答了“在稳定阶 段, 表面形貌依然在不断变化, 但摩擦因数可以保 持稳定” 的疑问。成功地将双高斯表面参数演化模型 应用于密封台架试验。

(6) 监测控制方面: 通过密封台架试验发现了 声发射信号与双高斯表面参数之间的相关性, 并依
此建立声发射模型。

综上所述, 今后可深入开展的机械密封双高斯 工作包括几个方面。

（1）双高斯分层理论起源于双工序表面，具有 实际的物理与工程意义。但工程实际中存在历经更 多加工工序而成的表面，该表面是形成多高斯分层 特征, 还是仅保留最后两道工序所致的双高斯特征, 有待涉及。鉴于摩擦磨损过程往往同时包含多种摩 擦磨损类型，上述多高斯拓展问题也同样适合于磨 损表面。

(2) 现有的双高斯润滑与接触研究, 都是机理 性质的模型建模工作，至多物性参数取自于机械密 封。后续, 可以基于现有的机理工作, 研究双高斯 分层特征对现有机械密封润滑与接触模型、性能的 影响。

（3）基于现有的双高斯润滑与接触机理模型, 开展摩擦磨损建模，进而应用于机械密封。

（4）现有双高斯声发射研究尚未构成完整体 系。后续, 可以探索将双高斯声发射模型应用于机 械密封, 分析密封端面的摩擦磨损状态, 监测密封 的运行状态。

\section{参 考 文 献}

[1] 顾永泉. 机械密封实用技术[M]. 北京：机械工业出版 社, 2001.

GU Yongquan. Practical technology of mechanical seal[M]. Beijing: China Machine Press, 2001.

[2] 王玉明, 杨惠霞, 姜南. 流体密封技术 [J]. 液压气动与 密封, 2004(3): 1-5.

WANG Yuming, YANG Huixia, JIANG Nan. Fluid seals technique[J]. Hydraulics Pneumatics \& Seals, 2004(3): $1-5$.

[3] 彭旭东, 王玉明, 黄兴, 等. 密封技术的现状与发展趋 势[J]. 液压气动与密封, 2009, 29(4): 4-11.

PENG Xudong, WANG Yuming, HUANG Xing, et al. State-of-the-art and future development of sealing technology[J]. Hydraulics Pneumatics \& Seals, 2009, 29(4): 4-11.

[4] HU S, BRUNETIERE N, HUANG W, et al. Continuous separating method for characterizing and reconstructing bi-Gaussian stratified surfaces[J]. Tribol. Int., 2016, 102: 454-462.

[5] HU S, HUANG W, BRUNETIERE N, et al. Truncated separation method for characterizing and reconstructing bi-Gaussian stratified surfaces[J]. Friction，2017， 5(1): 32-44.

[6] HU S, BRUNETIERE N, HUANG W, et al. Bi-Gaussian 
surface identification and reconstruction with revised autocorrelation functions[J]. Tribol. Int., 2017, 110: 185-194.

[7] HU S, HUANG W, LIU X, et al. Probe model of wear degree under sliding wear by Rk parameter set[J]. Tribol. Int., 2017, 109: 578-585.

[8] HU S, BRUNETIERE N, HUANG W, et al. Evolution of bi-Gaussian surface parameters of silicon-carbide and carbon-graphite discs in a dry sliding wear process[J]. Tribol. Int., 2017, 112: 75-85.

[9] HU S, BRUNETIERE N, HUANG $\mathrm{W}$, et al. The bi-Gaussian theory to understand sliding wear and friction[J]. Tribol. Int., 2017，114: 186-191.

[10] PEKLENIK J. New developments in surface characterisation and measurements by means of random process analysis[J]. Proc. IMechE., 1967, 182(11): 108-126.

[11] International Origanization for Standardzation. ISO 25178-2, surface texture: areal - part 2: Terms, definitions and surface texture parameters[S]. Switzerland: ISO, 2012.

[12] WHITEHOUSE D J. Assessment of surface finish profiles produced by multi-process manufacture[J]. Proc. IMechE. Part B: J. Eng. Manuf., 1985， 199(4): 263-270.

[13] MALBURG M C, RAJA J, WHITEHOUSE D J. Characterization of surface texture generated by plateau honing process[J]. CIRP Annals-Manufacturing Technology, 1993, 42(1): 637-639.

[14] SANNAREDDY H, RAJA J, CHEN K. Characterization of surface texture generated by multi-process manufacture[J]. Int. J. Mach. Tools Manuf., 1998, 38(5-6): 529-536.

[15] SHIMOMURA T, HIRABAYASHI H, NAKAJIMA T. A study of the relationship between frictional characteristics and surface condition of mechanical face seals[J]. Tribol. Trans., 1991, 34(4): 513-520.

[16] SHIMOMURA T, HIRABAYASHI H, NAKAJIMA T. A Study of the relationship between sealing performance and surface condition of mechanical face seals[J]. Tribol. Trans., 1992, 35(4): 659-666.

[17] LEEFE S E. "Bi-Gaussian” representation of worn surface topography in elastic contact problems[J]. Tribology Series 1998, 34: 281-290.

[18] LUBBINGE $\mathrm{H}$. On the lubrication of mechanical face seals[D]. Twente: University of Twente, 1999.

[19] MINET C, BRUNETIERE N, TOURNERIE B, et al. Analysis and modeling of the topography of mechanical seal faces[J]. Tribol. Trans., 2010， 53(6): 799-815.

[20] GRABON W , PAWLUS P, SEP J. Tribological characteristics of one-process and two-process cylinder liner honed surfaces under reciprocating sliding conditions[J]. Tribol. Int., 2010， 43: 1882-1892.

[21] PAWLUS P, MICHALCZEWSKI R, LENART A, et al. The effect of random surface topography height on fretting in dry gross slip conditions[J]. Proc. IMechE. Part J: J. Eng. Tribol., 2014, 228(12): 1374-1391.

[22] DZIERWA A, PAWLUS P, ZELASKO W. Comparison of tribological behaviors of one-process and two-process steel surfaces in ball-on-disc tests[J]. Proc. IMechE. Part J: J. Eng. Tribol., 2014, 228(10): 1195-1210.

[23] STAUFERT G. Characterization of random roughness profiles - A comparison of AR-modeling technique and profile description by means of commonly used parameters[J]. Annals CIRP, 1979, 28: 431-435.

[24] DEVRIES W R. Autoregressive time series models for surface profile characterization[J]. Annals CIRP, 1979, 28: $437-440$.

[25] WHITEHOUSE D J. The generation of two dimensional random surfaces having a specified function[J]. Annals CIRP, 1983, 32: 495-498.

[26] PATIR N. A numerical procedure for random generation of rough surfaces[J]. Wear, 1978，47(2): 263-277.

[27] BAKOLAS V. Numerical generation of arbitrarily oriented non-Gaussian three-dimensional rough surfaces[J]. Wear, 2004, 254(5-6): 546-554.

[28] HU Y, TONDER K. Simulation of 3-D random rough surface by 2-D digital filter and Fourier analysis[J]. Int. J. Mach. Tools Manuf., 1992, 32(1-2): 83-90.

[29] MAJUMDAR A, TIEN C. Fractal characterization and simulation of rough surfaces[J]. Wear, 1990, 136(2): 313-327.

[30] WU J. Simulation of rough surfaces with FFT[J]. Tribol. Int., 2000, 33: 47-58.

[31] WU J. Simulation of non-Gaussian surfaces with FFT[J]. Tribol. Int., 2004, 37: 339-346.

[32] ZAHOUANI H, VARGIOLU R, LOUBET J L. Fractal models of surface topography and contact mechanics[J]. Math. Comput. Model., 1998, 28(4-8): 517-534.

[33] PATRIKAR R M. Modeling and simulation of surface roughness[J]. Appl. Sur. Sci., 2004， 228(1-4): 213-220.

[34] ZOU M, YU B, FENG Y, et al. A Monte Carlo method for simulating fractal surfaces[J]. Physica A: Strtistical Mechanics and Its Applications, 2007, 386(1): 176-186.

[35] JOHNSON N L. Systems of frequency curves generated by method of translation[J]. Biometrika, 1949, 36 : 149-176.

[36] WATSON W, SPEDDING T A. The time series modelling of non-Gaussian engineering processes[J]. Wear, 1982, 
83(2): 215-231.

[37] HILL I D, HILL R, HOLDER R L. Fitting Johnson curves by moments[J]. Appl. Stat., 1976， 25(2): 180-189.

[38] NAU BS. Mechanical seal face materials[J]. Proc. IMechE. Part J: J. Eng. Tribol., 1997， 211(3): 165-183.

[39] SHIMOMURA T, KIRYU K, HIRABAYASHI H, et al. A study of the relationship between performance and surface characteristics of end face seals[J]. Lub. Eng., 1989, 45(12): 785-791.

[40] JACKSON R L, STREATOR J L. A multi-scale model for contact between rough surfaces[J]. Wear, 2006 , 261(11-12): 1337-1347.

[41] 杨惠霞, 顾永泉. 考虑表面雉度、波度和粗䊁度的两维 机械密封混合摩擦研究[J]. 流体机械, 1994, 22(5): 3-9. YANG Huixia, GU Yongquan. Study of two dimensional mixed friction of mechanical face seals considering surface taper, waviness and roughness[J]. Fluid Machinery, 1994, 22(5): 3-9.

[42] 冯向忠, 彭旭东. 表面粗䊁度对螺旋槽干式气体密封性 能的影响[J]. 润滑与密封, 2006(1): 20-22.

FENG Xiangzhon, PENG Xudong. Effect of surface roughness on performance of a spiral groove face seal[J]. Lubrication Engineering, 2006(1): 20-22.

[43] 彭旭东, 李纪云, 盛颂恩, 等. 表面粗粘度对螺旋槽干 式气体端面密封性能预测与结构优化的影响 [J]. 摩擦 学学报, 2007, 27(6): 567-572.

PENG Xudong, LI Jiyun, SHENG Songen, et al. Effect of surface roughness on performance prediction and geometric optimization of a spiral groove face seal[J]. Tribology, 2007, 27(6): 567-572.

[44] 魏龙, 顾伯勤, 冯飞, 等. 机械密封摩擦副端面形貌的 分形特性[J]. 石油化工高等学校学报, 2009, 22(2): 59-62.

WEI Long, GU Boqin, FENG Fei, et al. Fractal characteristics of end faces' topography in friction pair of mechanical seals[J]. Journal of Petrochemical University, 2009, 22(2): 59-62.

[45] 李双喜, 宋文博, 张秋翔, 等. 干式气体端面密封的开 启特性[J]. 化工学报, 2011，62(3): 766-772.

LI Shuangxi, SONG Wenbo, ZHANG Qiuxiang, et al. Opening characteristics of dry gas seal[J]. CIESC Journal, 2011, 62(3): 766-772.

[46] 陈汇龙, 刘志斌, 赵斌娟, 等. 表面粗粘度对多孔端面 机械密封膜压的影响[J]. 润滑与密封, 2014, 39(10): 16-21.

CHEN Long, LIU Zhibin, ZHAO Binjuan, et al. Effect of surface roughness on fluid film pressure of laser surface textured mechanical seal[J]. Lubrication Engineering, 2014, 39(10): 16-21.
[47] 魏龙, 顾伯勤, 刘其和, 等. 接触式机械密封端面平均 温度耦合计算方法 [J]. 化工学报, 2014, 65(9)： 3568-3575.

WEI Long, GU Boqin, LIU Qihe, et al. Average temperature coupling calculation method for end faces of contact mechanical seals[J]. CIESC Journal, 2014, 65(9): 3568-3575.

[48] JOHNSON KL. Contact mechanics[M]. Cambridge : Cambridge University Press, 1985.

[49] BOWDEN FP. Friction and lubrication of solids[M]. Oxford: Oxford University Press, 1954.

[50] GREENWOOD J A, WILLIAMSON J B P. Contact of nominally flat surfaces[J]. Proc. Royal Soc. A, 1966, 295(1442): 300-319.

[51] PULLEN J, WILLIAMSON J B P. On the plastic contact of rough surfaces[J]. Proc. Royal Soc. A , 1972 , 327(1569): 159-173.

[52] ABBOTT E J, FIRESTONE F A. Specifying surface quality: A method based on accurate measurement and comparison[J]. Mech. Eng., 1933, 55: 569-572.

[53] CHANG W R, ETSION I, BOGY D B. An elastic-plastic model for the contact of rough surfaces[J]. J. Tribol., 1987, 109(2): 257-263.

[54] WHITEHOUSE D J, ARCHARD J F. The properties of random surface of significance in their contact[J]. Proc. Royal Soc. A, 1970, 316(1524): 97-121.

[55] NAYAK P R. Random process model of rough surfaces in plastic contact[J]. Wear, 1973，26(3): 305-333.

[56] MCCOOL J I. Comparison of models for the contact of rough surfaces[J]. Wear, 1986, 107(1): 37-60.

[57] BHUSHAN B, DUGGER M T. Real contact area measurements on magnetic rigid disks[J]. Wear, 1990, 137(1): 41-50.

[58] MAJUMDAR A, BHUSHAN B. Role of fractal geometry in roughness characterization and contact mechanics of surfaces[J]. J. Tribol., 1990， 112(2): 205-216.

[59] SAYLES R S, THOMAS T R. Surface topography as a non-stationary random process[J]. Nature, 1978, 271(2): 431-434.

[60] MAJUMDAR A , BHUSHAN B. Fractal model of elastic-plastic contact between rough surfaces[J]. J. Tribol., 1991, 113(1): 1-11.

[61] YAN W, KOMVOPOULOS K. Contact analysis of elastic-plastic fractal surfaces[J]. J. Appl. Phys., 1998, 84(7): 3617-3624.

[62] 田红亮, 钟先友, 秦红玲, 等. 依据各向异性分形几何 理论的固定结合部法向接触力学模型 $[\mathrm{J}]$. 机械工程学 报，2013，49(21): 108-122.

TIAN Hongliang, ZHONG Xiangyou, QIN Hongling, 
et al. Normal contact mechanics model of fixed joint interface adopting anisotropic fractal geometrical theory[J]. Journal of Mechanical Engineering, 2013, 49(21): 108-122.

[63] 丁雪兴, 严如奇, 贾永磊. 基于基底长度的粗䊁表面分 形接触模型的构建与分析[J]. 摩擦学学报, 2014, 34(4): 341-347.

DING Xuexing, YAN Ruqi, JIA Yonglei. Construction and analysis of fractal contact mechanics model for rough surface based on base length[J]. Tribology, 2014, 34(4): 341-347.

[64] MORAG Y, ETSION I. Resolving the contradiction of asperities plastic to elastic mode transition in current contact models of fractal rough surfaces[J]. Wear, 2007, 262(5-6): 624-629.

[65] LUBRECHT AA, NAPEL W E, BOSMA R. Multigrid, an alternative method for calculating film thickness and pressure profiles in elastohydrodynamically lubricated line contacts[J]. J. Tribol.， 1986， 108(4): 551-556.

[66] LUBRECHT A A, NAPEL W E, BOSMA R. The influence of longitudinal and transverse roughness on the elastohydrodynamic lubrication of circular contacts[J]. J. Tribol., 1988, 110(3): 421-426.

[67] VENNER C H, NAPEL W E. Surface roughness effects in an EHL line contact[J]. J. Tribol., 1992, 114(3): 616-622.

[68] VENNER C H, LUBRECHT A A. Transient analysis of surface features in an EHL line contact in the case of sliding[J]. J. Tribol., 1994， 116(2): 186-193.

[69] VENNER C H, LUBRECHT A A. Numerical simulation of a transverse ridge in a circular EHL contact under rolling/sliding[J]. J. Tribol., 1994, 116(4): 751-761.

[70] AI X, CHENG S. The effects of surface texture on EHL point contacts[J]. J. Tribol., 1996, 118 (1): 59-66.

[71] VENNER C H, LUBRECHT A A. Numerical solution of the influence of waviness on the film thickness of a circular EHL contact[J]. J. Tribol., 1996, 118(1): 153-161.

[72] ZHU D, AI X. Point contact EHL based on optically measured three dimensional rough surfaces[J]. J. Tribol., 1997, 119(3): 375-384.

[73] HU Y, BARBER G C, ZHU D. Numerical analysis for the elastic contact of real rough surfaces[J]. Tribol. Trans., 1999, 42(3): 443-452.

[74] JIANG X, HUA D, CHENG S, et al. A mixed elastohydrodynamic lubrication model with asperity contact[J]. J. Tribol., 1999， 121(3): 481-491.

[75] HU Y, ZHU D. A full numerical solution to the mixed lubrication in point contacts[J]. J. Tribol., 2000， 122(1): $1-9$.

[76] HOOKE C J, VENNER C H. Surface roughness attenuation in line and point contacts[J]. Proc. IMechE. Part J: J. Eng. Tribol., 2000, 214(5): 439-444.

[77] WANG W, WANG H, HU Y, et al. A comparative study of the methods for calculation of surface elastic deformation[J]. Proc. IMechE. Part J: J. Eng. Tribol., 2003, 217(2): 145-153.

[78] WANG W, LIU Y, WANG H, et al. A computer thermal model of mixed lubrication in point contacts[J]. J. Tribol., 2004, 126(1): 162-170.

[79] WANG Q, ZHU D, CHENG S, et al. Mixed lubrication analyses by a macro-micro approach and a full-scale mixed EHL model[J]. J. Tribol., 2004, 126(1): 81-91.

[80] ZHU D, HU Y. A computer program package for the prediction of EHL and mixed lubrication characteristics, friction, subsurface stresses and flash temperatures based on measured 3-D surface roughness[J]. Tribol. Trans., 2001, 44(3): 383-390.

[81] ZHU D. On some aspects of numerical solutions of thin-film and mixed elastohydrodynamic lubrication[J]. Proc. IMechE. Part J: J. Eng. Tribol., 2007, 221(5): 561-579.

[82] BRUNETIERE N. An analytical approach of the thermoelastohydrodynamic behaviour of mechanical face seals operating in mixed lubrication[J]. Proc. IMechE. Part J: J. Eng. Tribol., 2010， 224(12): 1221-1233.

[83] MINET C, BRUNETIERE N, TOURNERIE B. A deterministic mixed lubrication model for mechanical seals[J]. J. Tribol., 2011， 133(4): 042203.

[84] MINET C, BRUNETIERE N, TOURNERIE B. On the lubrication of mechanical seals with rough surfaces: a parametric study[J]. Proc. IMechE. Part J: J. Eng. Tribol., 2012, 226(12): 1109-1126.

[85] NYEMECK A P, BRUNETIERE N, TOURNERIE B. A multiscale approach to the mixed lubrication regime: application to mechanical seals[J]. Tribol. Lett., 2012, 47(3): 417-429.

[86] BRUNETIERE N, WANG Q. Large-Scale simulation of fluid flows for lubrication of rough surfaces[J]. J. Tribol., 2013, 136(1): 011701.

[87] 魏龙, 顾伯勤, 冯秀. 机械密封摩擦副端面接触分形模 型[J]. 化工学报, 2009, 60(10): 2543-2548.

WEI Long, GU Boqin, FENG Xiu. Contact fractal model for friction faces of mechanical seals[J]. CIESC Journal, 2009, 60(10): 2543-2548.

[88] 魏龙，顾伯勤，孙见君，等. 机械密封摩擦副端面分形 维数的优化 $[\mathrm{J}]$. 化工学报, 2010, 61(1): 132-136. WEI Long, GU Boqin, SUN Jianjun, et al. Optimization of surface fractal dimension of friction pair in mechanical seals[J]. CIESC Journal， 2010，61(1): 132-136. 
[89] 周刚, 李晓钟, 朱维兵, 等. 机械密封环端面各向异性 分形接触分析[J]. 润滑与密封, 2013, 38(10): 55-59. ZHOU Gang, LI Xiaozhong, ZHU Weibing, et al. Contact analysis of anisotropic face on mechanical seal ring based on fractal model[J]. Lubrication Engineering, 2013, 38(10): 55-59.

[90] HARP S R, SALANT R F. Analysis of mechanical seal behavior during transient operation[J]. J. Tribol., 1998, 120(2): 191-197.

[91] GREEN I. A transient dynamic analysis of mechanical seals including asperity contact and face deformation[J]. Tribol. Trans., 2002, 45(3): 284-293.

[92] RUAN B. Numerical modeling of dynamic sealing behaviors of spiral groove gas face seals[J]. J. Tribol., 2002, 124(1): 186-195.

[93] HU S, HUANG W, LIU X, et al. Influence analysis of secondary O-ring seals in dynamic behavior of spiral groove gas face seals[J]. Chin. J. Mech. Eng., 2016, 29: 507-514.

[94] HU S, HUANG W, LIU X, et al. Application of fractal contact model in dynamic performance analysis of gas face seals[J]. Chin. J. Mech. Eng., 2018， 31: 27.

[95] SHAPIRO W, COLSHER R. Steady-state and dynamic analysis of a jet engine gas lubricated shaft seal[J]. ASLE Trans., 1974, 17(3): 190-200.

[96] MILLER B A, GREEN I. Numerical formulation for the dynamic analysis of spiral-grooved gas face seal[J]. J. Tribol., 2001, 123(2): 395-403.

[97] GREEN I, BARNSBY R M. A simultaneous numerical solution for the lubrication and dynamic stability of noncontacting gas face seals[J]. J. Tribol., 2001， 123(2): 388-394.

[98] GREEN I, BARNSBY R M. A parametric analysis of the transient forced response of noncontacting coned-face gas seals[J]. J. Tribol., 2002, 124(1): 151-157.

[99] ZIRKELBACK N, ANDRES L S. Effect of frequency excitation on force coefficients of spiral groove gas seals[J]. J. Tribol., 1999, 121(4): 853-861.

[100]ZIRKELBACK N. Parametric study of spiral groove gas face seals[J]. Tribol. Trans., 2000，43(2): 337-343.

[101]刘雨川. 端面气膜密封特性研究[D]. 北京: 北京航空航 天大学, 1999.

LIU Yuchuan. Research on sealing characteristics of end-face gas film[D]. Beijing: Beijing University of Aeronautics and Astronautics, 1999.

[102] RUAN B. A semi-analytical solution to the dynamic tracking of non-contacting gas face seals[J]. J. Tribol., 2002, 124(1): 196-202.

[103]刘雨川, 徐万孚, 王之栋, 等. 气膜端面密封角向摆动
自振稳定性[J]. 机械工程学报, 2002, 38(4): 1-6. LIU Yuchuan, XU Wanfu, WANG Zhiyue, et al. Stability of angular wobble self-excited vibrations for gas film face seal[J]. Chinese Journal of Mechanical Engineering, 2002, 38(4): 1-6.

[104]徐万孚, 刘雨川, 刘云飞, 等. 不同端面几何结构气膜 密封的刚度与自振稳定性[J]. 流体机械, 2001(11)： 22-25.

XU Wanfu, LIU Yuchuan, LIU Yunfei, et al. Stiffness and of self-excited vibration stability for gas film face seals with different geometries[J]. Fluid Machinery, 2001(11): 22-25.

[105]刘雨川, 徐万孚, 王之栋, 等. 端面气膜密封动力特性 系数的计算[J]. 清华大学学报, 2002, 42(2): 185-189. LIU Yuchuan, XU Wanfu, WANG Zhiyue, et al. Dynamic coefficients for gas film face seal[J]. Journal of Tsinghua University, 2002, 42(2): 185-189.

[106]徐万孚, 刘雨川, 李广宇, 等. 螺旋槽干运行非接触气 体密封的理论分析与试验[J]. 机械工程学报, 2003, 39(4): 22-25.

XU Wanfu, LIU Yuchuan, LI Guangyu, et al. Theoretical analysis and experimental investigation of sporal groove dry running noncontacting gas seals[J]. Chinese Journal of Mechanical Engineering, 2003, 39(4): 22-25.

[107]HU S, HUANG W, LIU X, et al. Stability and tracking analysis of gas face seals under low-parameter conditions considering slip flow[J]. J. Vibroeng., 2017, 19(3): 2126-2141.

[108]ELROD H G, MCCABE J T, CHU T Y. Determination of gas-bearing stability by response to a step-jump[J]. J. Lubr. Technol., 1967, 89(4): 493-498.

[109]MILLER B A, GREEN I. On the stability of gas lubricated triboelements using the step jump method[J]. J. Tribol., 1997, 119(1): 193-199.

[110] MILLER B A, GREEN I. Constitutive equations and the correspondence principle for the dynamics of gas lubricated triboelements[J]. J. Tribol., 1998, 120(2): 345-352.

[111] MILLER B A, GREEN I. Numerical techniques for computing rotordynamic properties of mechanical gas face seals[J]. J. Tribol., 2002， 124(4): 755-761.

[112] MILLER B A, GREEN I. Semi-Analytical dynamic analysis of spiral-grooved mechanical gas face seals[J]. J. Tribol., 2003, 125(2): 403-413.

[113] 徐辰. 压缩机用螺旋槽干气密封的动态特性分析与参 数研究[D]. 北京: 清华大学, 2014.

XU Chen. Analysis and parametric study on dynamic property of spiral groove dry gas seal in turbine compressor[D]. Beijing: Tsinghua University, 2014. 
[114]刘向锋, 徐辰, 黄伟峰. 基于半解析法的极端工况干气 密封动态特性研究与参数设计 [J]. 清华大学学报, 2014, 54(2): 223-228, 234.

LIU Xiangfeng, XU Chen, HUANG Weifeng. Analysis and parametric design of the dynamics of a dry gas seal for extreme operating conditions using a semi-analytical method[J]. Journal of Tsinghua University，2014， 54(2): 223-228, 234.

[115] XU C, HUANG W, LIU X. Tracking property analysis of a dry gas seal operating in low pressure condition[J]. Appl. Mech. Mater., 2014, 532: 367-373.

[116] ZHANG H, MILLER B A, LANDERS R G. Nonlinear modeling of mechanical has face seal systems using proper orthogonal decomposition[J]. J. Tribol., 2006, 128(4): 817-827.

[117] 张国渊, 赵伟刚, 问秀天, 等. 基于 POD 降阶模型的 非接触端面密封动态监测原理及仿真 [J]. 航空学报, 2012, 33(2): 354-361.

ZHANG Guoyuan, ZHAO Weigang, YAN Xiutian, et al. Principle and simulation for real-time monitoring of the non-contact face seal based on POD model[J]. Acta Aeronautica et Astronautica Sinica, 2012, 33(2): 354-361.

[118] ROSEN B G, OHLSSON R, THOMAS T R. Tribological implications of AFM measurements of cylinder bore microtopography[J]. Proceedings of the Conference on Atomic Scale Control of Surfaces and Interfaces, Brighton, UK, 1994.

[119] KUMAR R, KUMAR S, PRAKASH B, et al. Assessment of engine liner wear from bearing area curves[J]. Wear, 2000, 239(2): 282-286.

[120]DIMKOVSKI Z, ANDERBERG C, ROSEN B G, et al. Quantification of the cold worked material inside the deep honing grooves on cylinder liner surfaces and its effect on wear[J]. Wear, 2009, 267(12): 2235-2242.

[121]CORRAL I B, CALVET J V, SALCEDO M C. Use of roughness probability parameters to quantify the material removed in plateau-honing[J]. Int. J. Mach. Tools Manuf., 2010, 50(7): 621-629.

[122]DUNAEVSKY V V. Measurement of local microscopic wear[J]. J. Tribol., 1986, 108(1): 35-41.

[123]JENG Y, LIN Z, SHYU S. A microscopic wear measurement method for general surfaces[J]. J. Tribol., 2002, 124(4): 829-833.

[124]WANG Q, WANG Y, WANG H, et al. Experimental investigation on tribological behavior of several polymer materials under reciprocating sliding and fretting wear conditions[J]. Tribol. Int., 2016， 104: 73-82.

[125]MENEZES P L, KISHORE, KAILAS S V. Effect of roughness parameter and grinding angle on coefficient of friction when sliding of Al-Mg alloy over EN8 steel[J]. J. Tribol., 2006, 128(4): 697-704.

[126] MENEZES P L, KISHORE, KAILAS S V. Studies on friction and transfer layer: role of surface texture[J]. Tribol. Lett., 2006, 24(3): 265-73.

[127] MENEZES P L, KISHORE, KAILAS S V. Influence of surface texture and roughness parameters on friction and transfer layer formation during sliding of aluminium pin on steel plate[J]. Wear, 2009, 267(9-10): 1534-1549.

[128] MENEZES P L，KISHORE, KAILAS S V . Influence of roughness parameters and surface texture on friction during sliding of pure lead over 080 M40 steel[J]. Int. J. Adv. Manuf. Tech., 2009, 43(7-8): 731-743.

[129]MENEZES P L, KISHORE, KAILAS S V. Study of friction and transfer layer formation in copper-steel tribo-system: role of surface texture and roughness parameters[J]. Tribol. Trans., 2009, 52(5): 611-622.

[130]MENEZES P L, KISHORE, KAILAS S V, et al. Role of surface texture, roughness, and hardness on friction during unidirectional sliding[J]. Tribol. Lett., 2011, 41(1): 1-15.

[131] MENEZES P L, KISHORE, KAILAS S V, et al. Friction and transfer layer formation in polymer-steel tribo-system : role of surface texture and roughness parameters[J]. Wear, 2011, 271(9-10): 2213-2221.

[132] SEDLACEK M, PODGORNIK B, VIZINTIN J. Influence of surface preparation on roughness parameters, friction and wear[J]. Wear, 2009, 266(3-4): 482-487.

[133]SEDLACEK M, PODGORNIK B , VIZINTIN J. Correlation between standard roughness parameters skewness and kurtosis and tribological behavior of contact surfaces[J]. Tribol. Int., 2012, 48: 102-112.

[134] SEDLACEK M, PODGORNIK B, VIZINTIN J. Planning surface texturing for reduced friction in lubricated sliding using surface roughness parameters skewness and kurtosis[J]. Proc. IMechE. Part J: J. Eng. Tribol., 2012, 226(8): 661-667.

[135] PODGURSKY V, NISUMAA R, ADOBERG E, et al. Comparative study of surface roughness and tribological behavior during running-in period of hard coatings deposited by lateral rotating cathode arc $[\mathrm{J}]$. Wear, 2010, 268(5-6): 751-755.

[136]PODGURSKY V, ADOBERG E, SURZENKOV A. Dependence of the friction coefficient on roughness parameters during early stage fretting of $(\mathrm{Al}, \mathrm{Ti}) \mathrm{N}$ coated surfaces[J]. Wear, 2011，271(56): 853-858.

[137]PAWLUS P, GALDA L, DZIERWA A, et al. Abrasive wear resistance of textured steel rings[J]. Wear, 2009, 267(11): 1873-1882.

[138]LUGT P M， SEVERT R W M, FOGELSTROM J, et al. 
Influence of surface topography on friction, film breakdown and running-in in the mixed lubrication regime[J]. Proc. IMechE. Part J: J. Eng. Tribol., 2001, 215(6): 519-533.

[139]ETSION I. Experimental observation of the dynamic behavior of noncontacting coned-face mechanical seals[J]. ASLE Trans., 1984, 27(3): 263-270.

[140]KANTA M, JINNOUCHI Y, FUKAHORI M. Pumping action of aligned smooth face seals due to axial vibrations-theory[J]. J. Tribol., 1984, 106(3): 344-351.

[141]KANTA M, FUKAHORI M. Pumping action of aligned smooth face seals due to axial vibrations experiment[J]. J. Tribol., 1986, 108(1): 46-52.

[142]SALANT R F, MILLER A L, KAY P L, et al. Development of an electrically controlled mechanical seal[J]. Proceeding of the 11th International Conference on Fluid Sealing, BHRA, 1987: 576-595.

[143]HEILALA A J, KANGASNEIMI A. Adjustment and control of a mechanical seal against dry running and severe wear[J]. Proceeding of the 11th International Conference on Fluid Sealing, BHRA, 1987: 548-575.

[144]KOLLINGER R, NOSOWICZ J, RID U. Theoretical and experimental investigation into the running characteristics of gas-lubricated mechanical seals[J]. Proceeding of the 12th International Conference on Fluid Sealing, BHRA, 1989: 307-322.

[145]ETSION I, PALMOR Z J, HARARI N. Feasibility study of a controlled mechanical seal[J]. Lubr. Eng., 1991, 47(8): 621-625.

[146]WOLFF P. Experimental investigation of an actively controlled mechanical seal[D]. Atlanta: Georgia Institute of Technology, 1993.

[147]LEE A S, Green I. Physical modeling and data analysis of the dynamic response of a flexibly mounted rotor mechanical seal[J]. J. Tribol., 1995, 117(1): 130-135.

[148]ZOU M, GREEN I. Clearance control of a mechanical face seal[J]. Tribol. Trans., 1999, 42(3): 535-540.

[149]ZOU M, GREEN I. Dynamic simulation and monitoring of a non-contacting flexibly mounted rotor mechanical face seal[J]. Proc. IMechE. Part C: J. Mech. Eng. Sci., 2000, 214(9): 1195-1206.

[150]陈铭, 张秋翔, 蔡纪宁, 等. 气体端面密封试验设备 [J]. 流体机械, 2005, 33(2): 14-16.

CHEN Ming, ZHANG Qiuxiang, CAI Jining, et al. Experimental equipment of dry gas seal[J]. Fluid Machinery, 2005, 33(2): 14-16.

[151]张伟政. 干气密封非线性动力稳定性分析及其响应优 化[D]. 兰州: 兰州理工大学, 2012.

ZHANG Weizheng. Nonlinear dynamic stability analysis and response optimization in the system of dry gas seal[D]. Lanzhou: Lanzhou University of Technology, 2012.

[152]MIETTINEN J, SIEKKINEN V. Acoustic emission in monitoring sliding contact behavior[J]. Wear, 1995, 181-183: 897-900.

[153]BEGGAN C, WOULFE M, YOUNG P, et al. Using acoustic emission to predict surface quality[J]. Int. J. Adv. Manuf. Tech., 1999, 15(10): 737-742.

[154]KIM J S, KANG M C, RYU B J, et al. Development of an on-line tool-life monitoring system using acoustic emission signals in gear shaping[J]. Int. J. Manuf. Tech., 1999, 39(11): 1761-1777.

[155]CHOUDHURY A, TANDON N. Application of acoustic emission technique for the detection of defects in rolling element bearings[J]. Tribol. Int., 2000, 33: 39-45.

[156] AL-GHAMD A M， MBA D. A comparative experimental study on the use of acoustic emission and vibration analysis for bearing defect identification and estimation of defect size[J]. Mech. Syst. Signal Pr., 2006, 20(7): 1537-1571.

[157]HE Y, ZHANG X, FRISWELL M I. Defect diagnosis for rolling element bearings using acoustic emission[J]. J. Vib. Acoust., 2009, 131(6): 061012.

[158]TAN C K, MBA D. Correlation between acoustic emission activity and asperity contact during meshing of spur gears under partial elastohydrodynamic lubrication[J]. Tribol. Lett., 2005, 20(1): 63-67.

[159]CHAARI F, BACCAR W, ABBES M S, et al. Effect of spalling or tooth breakage on gear mesh stiffness and dynamic response of a one-stage spur gear transmission[J]. Eur. J. Mech. A-Solid, 2008， 27(4): 691-705.

[160] SHARMA R B, PAREY A, TANDON N. Modelling of acoustic emission generated in involute spur gear pair[J]. J. Sound. Vib., 2017, 393: 353-373.

[161]ORCUTT F K. An investigation of the operation and failure of mechanical face seals[J]. J. Lubr. Tech., 1969, 91(4): 713-725.

[162] MIETTINEN J, SIEKKINEN V. Acoustic emission in monitoring sliding contact behavior[J]. Wear, 1995, 181-183: 891-900.

[163]HOLENSTEIN A P, INNOTEC S, FLEPP B. Diagnosis of mechanical seals in large pumps[J]. Sealing Technology, 1996, (33): 9-12.

[164] MBA D, ROBERTS T, TAHERI E, et al. Application of acoustic emission technology for detecting the onset and duration of contact in liquid lubricated mechanical seals[J]. Insight : Non-Destructive Testing and Condition Monitoring, 2006, 48(8): 486-487.

[165]FAN Y. The condition monitoring of mechanical seals 
using acoustic emissions[D]. Manchester: The University of Manchester, 2007.

[166] HUANG W, LIN Y, GAO Z, et al. An acoustic emission study on the starting and stopping processes of a dry gas seal for pumps[J]. Tribol. Lett., 2013， 49(2): 379-384.

[167]HUANG W, LIN Y, LIU Y, et al. Face rub-impact monitoring of a dry gas seal using acoustic emission[J]. Tribol. Lett., 2013, 52(2): 253-259.

[168] 张尔卿, 傅攀, 陈㑆, 等. 机械密封端面接触状态的声 发射监测方法[J]. 润滑与密封, 2014, 39(8): 8-13, 34. ZHANG Erqing, FU Pan, CHEN Kan, et al. Mechanical fluid seal end face contact state monitoring based on acoustic emission[J]. Lubrication Engineering, 2014, 39(8): 8-13, 34.

[169]林志斌, 傅攀, 张尔卿, 等. 基于声发射和小波神经网 络的机械密封状态分类新方法[J]. 润滑与密封, 2014, 39(9): 40-45.

LIN Zhibin, FU Pan, ZHANG Erqing, et al. A new classifying method for mechanical seal condition based on acoustic emission and wavelet neural network[J]. Lubrication Engineering, 2014，39(9): 40-45.

[170]李晓晖, 傅攀, 曹伟青, 等. 机械密封端面接触状态的 声发射监测研究 $[\mathrm{J}]$. 振动与冲击, 2016, 35(8): 84-89. LI Xiaohui, FU Pan, CAO Weiqing, et al. The study of acoustic emission monitoring for contact state of seal end faces[J]. Journal of Vibration and Shock, 2016, 35(8): 84-89.

[171]THOMAS T R. Computer simulation of wear[J]. Wear, 1972, 22(1): 84-90.

[172]KING T G, WATSON W, STOUT K J. Modelling the micro-geometry of lubricated wear[C]// Proceedings of the 4th Leeds-Lyon Symposium on Tribology, London, MEP, 1978, 333-343.

[173]HU S, BRUNETIERE N, HUANG W, et al. Stratified effect of continuous bi-Gaussian rough surface on lubrication and asperity contact[J]. Tribol. Int., 2016， 104: 328-341.

[174]HU S， BRUNETIERE N, HUANG W, et al. Stratified revised asperity contact model for worn surfaces[J]. J. Tribol., 2017, 139: 021403.
[175]WHITEHOUSE D J. Surfaces - a link between manufacture and function[J]. Proc. IMechE., 1978, 192(1): 179-188.

[176]WILLIAMSON J P B. Microtopography of surfaces[J]. Proc. IMechE., 1967, 182(11): 21-30.

[177] International Origanization for Standardzation. ISO 13565-3, surface texture: profile method; Surfaces having stratified functional properties - Part 3: Height characterization using the material probability curve[S]. Switzerland: ISO, 1998.

[178]PAWLUS P. Simulation of stratified surface topographies[J]. Wear, 2008, 264(5-6): 457-463.

[179]ZELASKO W, PAWLUS P, DZIERWA A, et al. Experimental investigation of plastic contact between a rough steel surface and a flat sintered carbide surface[J]. Tribol. Int., 2016, 100: 141-151.

[180]PAWLUS P, ZELASKO W, REIZER R, et al. Calculation of plasticity index of two-process surfaces[J]. Proc. IMechE. Part J: J. Eng. Tribol., 2017， 231(5): 572-582.

[181]胡松涛. 基于双高斯分层表面理论的密封摩擦磨损研 究[D]. 北京: 清华大学, 2017.

HU Songtao. Study on seal tribological properties using a bi-gaussian stratified surface theory[D]. Beijing: Tsinghua University, 2017.

[182] HU S, HUANG W， SHI X, et al. Bi-Gaussian stratified effect of rough surfaces on acoustic emission under a dry sliding friction[J]. Tribol. Int., 2018， 119: 308-315.

作者简介: 胡松涛, 男, 1989 年出生, 博士后。主要研究方向为表面科 学、摩擦学、润滑与密封。

E-mail: hsttaotao@sjtu.edu.cn

黄伟峰, 男, 1978 年出生, 博士, 副研究员。主要研究方向为机械密封。 E-mail: huangwf@tsinghua.edu.cn

史熙(通信作者), 男, 1974 年出生, 博士, 教授。主要研究方向为界面 强度及摩擦学设计。

E-mail: xishi@sjtu.edu.cn

彭志科, 男, 1974 年出生, 博士, 教授。主要研究方向为信号分析、故 障诊断。

E-mail: z.peng@sjtu.edu.cn

刘向锋, 男, 1961 年出生, 博士, 教授。主要研究方向为机械设计、机 械密封。

E-mail: liuxf@tsinghua.edu.cn 